\title{
PRODUÇÃO CIENTÍFICA BRASILEIRA SOBRE SOFRIMENTO PSÍQUICO E DEPRESSÃO DA EQUIPE DE ENFERMAGEM NA EMERGÊNCIA
}

\author{
BRAZILIAN SCIENTIFIC PRODUCTION ON PSYCHIC SUFFERING \\ AND DEPRESSION IN NURSES WORKING IN THE \\ EMERGENCY DEPARTMENT
}

\section{Michele Antunes}

Email para correspondência: micheleantunes@gmail.com Enfermeira. Doutoranda em Informática na Educação na Universidade Federal do Rio Grande do Sul. Professora na Universidade Feevale. Nova Hamburgo, Rio Grande do Sul, Brasil.

RESUMO I Os profissionais da enfermagem que trabalham nos setores de emergência e urgência estão mais suscetíveis ao sofrimento psíquico e à depressão, pois o ambiente profissional com as características e organização do trabalho de implica em constantes situações estressoras. Objetivo: identificar a produção científica brasileira sobre as causas do sofrimento psíquico e depressão da equipe de enfermagem que atua nos serviços de emergência. Metodologia: revisão integrativa de caráter exploratório e descritivo nas bases de dados Literatura LatinoAmericano e do Caribe em Ciências da Saúde (LILACS) e o repositório Scientific Electronic Library Online (Scielo), com as palavras-chave: "sofrimento psíquico" e "emergência" e "depressão", no período de 2010 a 2016, resultando em uma amostra de sete estudos. Resultados: causas da depressão e sofrimento psíquico são a violência sofrida no seu ambiente de trabalho, os atendimentos emergenciais e os fatores organizacionais como $\circ$ ambiente físico, normas institucionais, burocracia, comunicação, autonomia, recompensas, segurança) que com outros fatores como os pessoais e do trabalho, a dificuldade em separar o que é estresse físico ou psicológico e a vulnerabilidade psíquica a qual estes profissionais estão expostos, fatos estes que que podem estar associadas a sintomas de sofrimento psíquico e depressão. Conclusão: é necessário a elaboração de ações de prevenção e promoção da saúde do trabalhador que atuam na emergência, principalmente com foco multiprofissional. Ações como a elaboração de grupos de discussão e a prática da escuta qualificada com o auxílio de um psicólogo, poderiam ser implementadas.

\begin{abstract}
I Nursing professionals that work in emergency and urgency departments are more susceptible to psychological distress and depression since the professional environment, its characteristics and organization of work imply in constant stressful situations. Objective: identify the Brazilian scientific literature on the causes of psychological distress and depression in the nursing team working in the emergency services. Method: integrative review of an exploratory and descriptive character was carried out in the databases Latin American and Caribbean Health Sciences (LILACS) and Scientific Electronic Library Online repository (Scielo), with the keywords "mental suffering", "emergency" and "depression", including papers published from 2010 to 2016 , resulting in a sample of seven studies. Results: causes of depression and psychic suffering are violence in the workplace, emergency care and organizational factors such as physical environment, institutional norms, bureaucracy, communication, autonomy, rewards, safety) than with other factors such as personal And work, the difficulty in separating what is physical or psychological stress and the psychic vulnerability to which these professionals are exposed, facts that may be associated with symptoms of psychic suffering and depression. Conclusion: it was concluded that the development of prevention and promotion of occupational health in emergency departments is necessary and needs to be multifocal. Actions such as the development of discussion groups and the practice of qualified hearing with the help of a psychologist could be implemented.
\end{abstract}

Palavras-chave: Assistência ambulatorial. Recursos Humanos de Enfermagem. Esgotamento Profissional. 


\section{INTRODUÇÃO}

O exercício profissional no âmbito hospitalar é marcado por múltiplas exigências: lidar com dor, sofrimento, morte e perdas, a que se somam as condições desfavoráveis de trabalho e a baixa remuneração, fatores que, em conjunto, propiciam a emergência de estresse e burnout, termo criado para descrever o desgaste físico e psíquico de profissionais que lidam, no exercício de suas funções, com altos níveis de envolvimento emocional'.

Os profissionais da enfermagem que estão lotados nos setores de emergência e urgência são vencedores quando se fala em estresse, pois estão ligados diretamente à doença, a dor e a morte. Convivendo continuamente com inúmeros sentimentos que os levam ao estresse e desgaste físico e mental, tendo que prestar assistência com qualidade num espaço físico que muitas vezes é desconfortável.

ambiente hospitalar, por si só, também pode ser considerado como um fator estressor, uma vez que possui condições de insalubridade $e$ periculosidade em relações a outros tipos de serviços que são prestados por esses profissionais ${ }^{2}$. Estes relacionaram sofrimento psíquico pessoal ao ambiente de trabalho, principalmente à falta de suporte e condições negativas de trabalho. $O$ ambiente profissional com as características e organização do trabalho de Enfermagem abarca constantes situações estressoras ${ }^{3}$.

Segundo autores ${ }^{4}$, os trabalhadores que atuam na emergência podem sentir desgastes físico, emocional e estresse. Isso, porque o ambiente em que atua, em conjunto com uma equipe multiprofissional, exige resultados imediatos do processo de trabalho, responsabilizando-o pela qualidade do atendimento ao paciente. Além disso, esses profissionais têm de suportar continuamente o risco iminente de morte, onde a complexidade dos cuidados que prestam aliados aos fatores de âmbito pessoal desencadeia o estresse e o sofrimento psíquico.

Em estudos prévios que almejaram identificar a depressão em trabalhadores de emergência, identificou que mais de $90 \%$ dos profissionais de emergência adoecidos por depressão e que a maioria dos enfermeiros, não percebia $\circ$ próprio sofrimento psíquico, não o relacionava às condições de trabalho e acreditava não haver influência desse sofrimento na assistência prestada ${ }^{5}$. Em outro estudo os trabalhadores de enfermagem informaram que - desgaste psíquico sofrido com a própria natureza do trabalho (cuidar de vidas adoecidas) é menos impactante sobre a qualidade de vida do que o desgaste, originado pelas situações e relações sociais presentes no trabalho ${ }^{6}$.

A identificação dos elementos estressores em atendimentos de urgência e emergência, corresponde a um dos grandes agentes de transformação deste contexto. Produzindo ações no sentido da valorização dos aspectos humanos e profissionais desses sujeitos. Nesse sentido, cabe destacar a importância do reconhecimento dos estressores e de seus efeitos sobre o organismo, para que sejam adotadas medidas de enfrentamento a fim de evitar distúrbios psicológicos e fisiológicos ${ }^{7}$.

A relevância neste estudo consiste no fato de que a partir da identificação do sofrimento psíquico dos profissionais de enfermagem na emergência, podese sugerir a implementação de ações de redução dos agentes o estão causando, proporcionando assim, a sua redução e melhorando a qualidade de vida do trabalhador.

Diante do exposto, o objetivo deste estudo foi o de identificar a produção científica brasileira sobre as causas do sofrimento psíquico e depressão da equipe de enfermagem que atua nos serviços de emergência. Os objetivos específicos foram o de analisar quais os fatores que podem desencadeálos, assim como o de analisar as causas deste.

Neste contexto surgem algumas inquietações, tais como o que causa o sofrimento psíquico e a depressão da equipe de enfermagem nos serviços de emergência? São os recursos materiais ou as relações interpessoais entre os profissionais? $O$ que pode desencadear este processo de sofrimento ou de depressão? 


\section{MÉTODOS}

Trata-se de uma revisão integrativa de caráter exploratório e descritivo desenvolvida em cinco etapas: formulação do problema, coleta dos dados, avaliação dos dados coletados, análise e interpretação dos dados, e apresentação dos resultados ${ }^{8}$.

A formulação do problema se caracterizou pelas questões norteadoras: quais são as causas do sofrimento psíquico da equipe de enfermagem nos serviços de emergência? Quais as causas e prejuízos para a equipe e paciente? Para a coleta dos dados definiu-se as bases de dados Literatura LatinoAmericano e do Caribe em Ciências da Saúde (LILACS) e o repositório Scientific Electronic Library Online (Scielo). Para a realização da busca, foram utilizadas as palavras-chave: "sofrimento psíquico" e "emergência". Os critérios de inclusão para a seleção da amostra foram: artigos publicados em português, publicados $e$ indexados nas referidas bases de dados, no período de 2010 a 2016, pesquisas qualitativas, quantitativas, quali-quantitativas e que tratassem da temática em estudo. Os critérios de exclusão foram: teses, dissertações, manuais, anais de eventos científicos, artigos sem acesso ao texto completo, indisponíveis online, e que não tratassem da temática.

Para a realização do registro das informações de modo a organizá-las de acordo com a questão norteadora do estudo, elaborou-se um instrumento para o que compreendia dados de identificação dos artigos (título, periódico, ano), objetivo/questão de investigação dos estudos, metodologia, resultados e as limitações/recomendações.

A apresentação dos resultados foi realizada por meio de quadros e tabelas. Ressalta-se ainda que - compromisso com os aspectos éticos consistiu na citação dos autores dos estudos analisados.

\section{RESULTADOS E DISCUSSÃO}

Identificaram-se 83 trabalhos na LILACS e no Scielo. Destes, 35 estavam disponíveis na íntegra. Estes tiveram seus resumos lidos separadamente, e destes, 24 foram excluídos por não contemplarem $\circ$ foco do estudo. Das 11 pesquisas restantes, três estudos estavam presentes em ambas as bases de dados, sendo contabilizados somente uma vez. Um artigo foi excluído por se tratar de sofrimento psíquico em médicos. Portanto, a amostra constituiu-se de sete artigos científicos. Estes foram lidos na íntegra e compuseram a amostra desta pesquisa.

Dos sete estudos que constituíram a amostra (Tabela 1), todos são resultados de pesquisa, sendo $03(42,8 \%)$ estudos transversais com abordagem quantitativa, $01 \quad(14,2 \%)$ estudo transversal qualitativo, $01 \quad(14,2 \%)$ transversal quantiqualitativo, 01 (14,2\%) estudo descritivo quantitativo e $01(14,2 \%)$ estudo descritivo qualitativo. No que se refere aos periódicos em que os estudos foram publicados, destaca-se que $02(28,5 \%)$ pesquisas foram publicados na Acta Paulista de Enfermagem e $02(28,5 \%)$ foram publicados na Revista da Escola de Enfermagem da USP. Com relação ao ano de publicação, o de 2011 foi o período prevalente, compreendendo uma amostra de $3(42,8 \%)$.

Após a leitura, análise e síntese do conteúdo dos artigos, identificou-se que o objetivo dos estudos foram o de identificar a associação de dados sociodemográficos, ocupacionais e econômicos em trabalhadores de enfermagem com a presença de sintomas de estresse", analisar os preditores da síndrome de burnout apresentados por enfermeiros ${ }^{10}$, conhecer suas vivências acerca do contexto laboral de trabalhadores de enfermagem ${ }^{11}$, evidenciar as vivências subjetivas dos trabalhadores em sua relação com a precarização do trabalho e as estratégias desenvolvidas para lidar com 0 sofrimento ${ }^{12}$, caracterizar os sintomas de estresse ${ }^{2}$, identificar a violência sofrida pelos trabalhadores da equipe de saúde e a sua associação com burnout e transtornos psíquicos menores ${ }^{13}$ e verificar se enfermeiros do serviço hospitalar de emergência apresentavam sintomas depressivos, identificar fatores intervenientes e analisar percepção sobre - sofrimento psíquico e influência na assistência prestada $^{5}$. A partir destes achados, percebe-se que os estudo buscam não somente identificar a presença do sofrimento psíquico e da depressão, mas também relaciona-los com a sua etiologia, assim como, buscaram investigar as estratégias utilizadas pelos profissionais para que possam estar 
gerenciando este sofrimento.

No que se refere a identificação do sofrimento psíquico e depressão na equipe de enfermagem que atua no cenário da emergência, os dados apontam para que a maioria dos profissionais vivenciam e apresentam sintomas de sofrimento, e que estes são causados pelo trabalho neste cenário (Tabela 1).

O que se refere as causa do sofrimento psíquico e da depressão, os estudos evidenciam que podem estar pode estar mais relacionado com fatores organizacionais (ambiente físico, mudanças organizacionais, normas institucionais, burocracia, comunicação, autonomia, recompensas, segurança) que com outros fatores como os pessoais (idade, sexo, nível educacional, filhos, lazer, etc.), do trabalho (tipo de ocupação, tempo de profissão, tempo de instituição, trabalho por turnos ou noturno, sobrecarga, tipo de cliente, etc.) e/ou achados sóciodemográficos ${ }^{10}$. Já em outra pesquisa, o sofrimento está relacionado com as limitações que encontravam para realizar o trabalho da forma como as regras de seu ofício lhes determinavam ${ }^{12}$. Este sofrimento pode ser causado pelo somatório de fatores organizacionais e de fatores que são inerentes ao próprio trabalho no cenário da emergência, conforme a Figura 1.

Ressalta-se ainda que 0 estresse emocional sempre apareciam associadas após atendimentos emergenciais, o que leva a crer que existe uma grande dificuldade em se separar o estresse físico do psíquico ${ }^{2}$. Os profissionais possuem dificuldade em delimitar o estresse que é desencadeado pelo atendimento a um paciente grave e crítico, da sobrecarga física que este paciente exige e desencadeia na equipe de saúde.

Em outro estudo o sofrimento constatado no trabalho de campo pode ser considerado indicador de vulnerabilidade e risco à saúde para as trabalhadoras de enfermagem ${ }^{1}$. Os trabalhadores de saúde sofrem violência em seu ambiente de trabalho e a essa exposição deixa-os mais submetidos a diferentes formas de perpetração da violência experimentam ainda mais esses agravos ${ }^{13}$.

No que se refere a prevalência da depressão, mais de $90 \%$ dos profissionais de emergência adoecidos por depressão. Estes dados evidenciam a necessidade de reflexões e intervenções com a finalidade de possibilitar melhoria das condições de trabalho e consequente melhora na saúde mental desses profissionais ${ }^{5}$.

No que se refere às limitações evidenciadas pelos estudos, identifica-se o foco exclusivo dado aos trabalhadores de enfermagem, sendo que outros profissionais da equipe de saúde também experimentam, de alguma forma, as repercussões do processo de trabalho no pronto-socorro com esses trabalhadores $^{11}$. Vê-se a necessidade de realizar estudos com uma abordagem multiprofissional para identificar e elaborar ações com foco na promoção da saúde do trabalhador que atua em um contexto de emergência.

\section{CONCLUSÃO}

Os achados deste estudo permitiram identificar que as causas da depressão e sofrimento psíquico são a violência sofrida no seu ambiente de trabalho, os atendimentos emergenciais e os fatores organizacionais como o ambiente físico, normas institucionais, burocracia, comunicação, autonomia, recompensas, segurança) que com outros fatores como os pessoais (idade, sexo, nível educacional, filhos, lazer, etc.), do trabalho (tipo de ocupação, tempo de profissão, tempo de instituição, trabalho por turnos ou noturno, sobrecarga, tipo de cliente), a dificuldade em separar o que é estresse físico ou psicológico e a vulnerabilidade psíquica a qual estes profissionais estão expostos, fatos estes que que podem estar associadas a sintomas de sofrimento psíquico e depressão.

Acredita-se que os resultados deste estudo corroboram com a vivência de atuação dos profissionais de atuam na emergência, tendo em vista que constantemente assistimos pacientes com um nível de gravidade elevado e que demandam de muitos recursos materiais e que exigem elevada carga de trabalho da equipe de enfermagem. Soma-se a isso, as normas institucionais e a burocracia exigida para que o perfil de qualidade assistencial seja executado, apesar de muitas vezes, a própria 
instituição não oferecer estrutura para executá-lo.

Ressalta-se ainda que estudos que tratam desta temática no contexto brasileiro ainda se apresentam em pouca quantidade evidenciando uma lacuna de conhecimento na área da saúde do trabalhador que necessita ser explorada. Tendo em vista que a identificação destes sintomas de sofrimento psíquico e de depressão são essenciais para que sejam elaboradas estratégias e ações de prevenção e promoção da saúde do trabalhador que tua na emergência. Dentre estas, destaca-se a atuação do Setor de Medicina do Trabalho, na qual poderia estar realizando a avaliação periódica da qualidade do trabalho destes profissionais, desenvolvendo grupos de apoio e a busca ativa daqueles que poderiam estar mais suscetíveis a depressão e sofrimento psíquico, pois acredita-se que a prevenção e o tratamento deveriam ter uma abordagem multidisciplinar, na qual enfermeiro, psicólogo e médico, poderiam estar intervindo para que haja uma melhora na qualidade do trabalho e na qualidade de vida destes.

Como recomendação, destaca-se a realização de mais pesquisas com foco multiprofissional, que tenham como foco a saúde do trabalhador que está sujeito a apresentar sofrimento e sintomas depressivos, pois o que consta na literatura brasileira ainda é um quantitativo incipiente.

\section{CONFLITOS DE INTERESSES}

Nenhum conflito financeiro, legal ou político envolvendo terceiros (governo, empresas e fundações privadas, etc.) foi declarado para nenhum aspecto do trabalho submetido (incluindo mas não limitandose a subvenções e financiamentos, conselho consultivo, desenho de estudo, preparação de manuscrito, análise estatística, etc).

\section{REFERÊNCIAS}

1. Avellar LZ, Iglesias A, Valverde PF. Sofrimento psíquico em trabalhadores de enfermagem de uma unidade de oncologia. Revista Psicologia em Estudo. 2007;1 2(3):475-481

2. Farias SMC, Teixeira OLC, Moreira W, Oliveira

MAF, Pereira MO. Caracterização dos sintomas físicos de estresse na equipe de pronto atendimento. Rev Esc Enferm USP. 2011 ;45(3):722-729. doi: 10.1590/S0080$\underline{62342011000300025}$

3. Pisanti $R$, van der Doef $M$, Maes S, Lazzari $D$, Bertini $M$. Job characteristics, organizational conditions, and dustress/ well being among italian and dutch nurses: a cross-national comparison. Int J Nurs Stud. 2011 ;48(7):829-37

4. Bezerra FN, Silva TM, Ramos VP. Estresse ocupacional dos enfermeiros de urgência e emergência: Revisão Integrativa da Literatura. Acta paul enferm. 2012;25(spe2): 151-156

5. Oliveira FP, Mazzaia MC, Marcolan JF. Sintomas de depressão e fatores intervenientes entre enfermeiros de serviço hospitalar de emergência. Acta paul enferm. 2015;28(3):209-15. doi: 10.1590/1982-0194201500036

6. Mininel VA, Baptista PCP, Felli VEA. Cargas psíquicas e processos de desgaste em trabalhadores de enfermagem de hospitais universitários brasileiros. Rev Lat Am Enfermagem. 2011 ; 19(2). doi: 10.1590/S0104-11692011000200016

7. Melo MV, Silva TP, Novais ZG, Mendes MLM. Estresse dos profissionais de saúde nas unidades hospitalares de atendimento em urgência e emergência. Cadernos de Graduação - Ciências Biológicas e da Saúde Facipe. $2013 ; 1(2): 35-42$

8. Cooper HM. The integrative research review: a systematic approach. Beverly Hills: Sage; 1984

9. Seleghim MR, Mombelli MA, Oliveira MLF, Waidman MAP, Marcon SS. Sintomas de estresse em trabalhadoras de enfermagem de uma unidade de pronto socorro. Rev Gaucha Enferm. 2012;33(3):165-173. doi: 10.1590/S198314472012000300022

10. França SPS, Aniceto EVS, DeMartino MMF. Preditores da Síndrome de Burnout em enfermeiros de serviços de urgência pré-hospitalar. Acta paul enferm. $2012 ; 25(1)$ :68-73. doi: $10.1590 /$ S0103-21002012000100012

11. Pai DD, Lautert L. Sofrimento no trabalho de Enfermagem. Escola Anna Nery. 2011 ; 15(3):524-530. doi: 10.1590/ $\underline{\text { s1414-81452011000300012 }}$

12. Silva NM, Muniza HP. Vivências de trabalhadores em contexto de precarização: um estudo de caso em serviço de emergência de hospital universitário. Estud Pesqui Psicol. $2011 ; 11(3): 821-40$

13. Pai DD, Lautert L, Souza SBC, Marziale MHP, Tavares JP. Violência, burnout e transtornos psíquicos menores no trabalho hospitalar. Rev Esc Enferm USP. 2015;49(3):460468. doi: $10.1590 /$ S0080-623420150000300014 